\title{
Residual mass in five-dimensional fermion formulations
}

\section{S. Hashimoto*}

High Energy Accelerator Research Organization (KEK), Tsukuba 305-0801, Japan and School of High Energy Accelerator Science, The Graduate University for Advanced Studies

(Sokendai), Tsukuba 305-0801, Japan

E-mail: shoji.hashimotodkek.jp

\section{S. Aoki}

Yukawa Institute for Theoretical Physics, Kyoto University, Kyoto 606-8502, Japan

\section{G. Cossu}

High Energy Accelerator Research Organization (KEK), Tsukuba 305-0801, Japan

\section{H. Fukaya}

Department of Physics, Osaka University, Toyonaka 560-0043, Japan

\section{T. Kaneko}

High Energy Accelerator Research Organization (KEK), Tsukuba 305-0801, Japan and School of High Energy Accelerator Science, The Graduate University for Advanced Studies (Sokendai), Tsukuba 305-0801, Japan

\section{J. Noaki}

High Energy Accelerator Research Organization (KEK), Tsukuba 305-0801, Japan

\section{P. A. Boyle}

SUPA, School of Physics, The University of Edinburgh, Edinburgh EH9 3JZ, UK

Using a general five-dimensional representation of the Ginsparg-Wilson fermion, we study the residual chiral symmetry violation and numerical costs of various fermion formulations including the domain-wall and overlap fermions as special cases.

31st International Symposium on Lattice Field Theory - LATTICE 2013

July 29 - August 3, 2013

Mainz, Germany

\footnotetext{
*Speaker.
} 


\section{Chiral symmetry on the lattice}

Chiral symmetry on the lattice can be realized by a Dirac operator that satisfies the GinspargWilson relation [四]. This class of the Dirac operator is represented by the form [వ]

$$
D=\frac{M_{0}}{a}\left[1-\gamma_{5} \operatorname{sgn}\left(a H_{K}\left(-M_{0}\right)\right)\right], \quad H_{K}\left(-M_{0}\right) \equiv \gamma_{5} D_{K}\left(-M_{0}\right),
$$

with some kernel operator $D_{K}$ and a matrix sign function. The parameter $M_{0}$ also appears in the definition of $D_{K}$ as a (negative) mass term; its value is typically in the range between 1.0 and 1.8 , chosen such that the number of near-zero modes of $H_{K}$ is minimized. In practice, exactly calculating the sign function is extremely costly for a large matrix $H_{K}$, and one has to introduce some approximation of it, such as the polynomial or the rational function. Such approximation becomes more difficult for the eigenvalues of $H_{K}$ near zero, where sgn is singular.

In the overlap fermion formulation the kernel operator $H_{K}$ is the hermitian Wilson-Dirac operator $D_{W}$, while the standard domain-wall fermion has the kernel of the form $H_{T} \equiv H_{W} /\left(2+D_{W}\right)$. For the sign function approximation one typically uses the optimal rational approximation (Zolotarev) for the overlap fermion, and the polar or hyperbolic tangent (Tanh) approximation for domain-wall, but other choices or combinations can give an equally good implementation of the representaion (III). The practical question is therefore how precisely one can approximate the sign function, and it determines how well the resulting operator preserves chiral symmetry.

In this work we test various choices of the kernel and approximation by examining their residual mass and numerical costs. The residual mass is a measure of the violation of the GinspargWilson relation. The five-dimensional construction of $D$ is useful as it allows us to easily switch from one choice to another by simply setting parameters. In the next section we briefly describe the formulation we took. Numerical results are presented in the following sections.

\section{Generalized 5D representation}

We follow the five-dimensional (5D) representaions summarized by Edwards and Heller [B]] (for the original ideas, see the references therein). In this generalized 5D representation, one defines a 5D fermion field $\psi_{5}$ and a lattice action $S_{G S W}=\sum_{x} \bar{\psi}_{5} D_{G D W}^{5} \psi_{5}$ with a $5 \mathrm{D}$ operator

$$
D_{G D W}^{5}=\left(\begin{array}{cccccc}
\left(D_{-}^{1}\right)^{-1} D_{+}^{1} & -P_{-} & 0 & \cdots & 0 & m P_{+} \\
-P_{+} & \left(D_{-}^{2}\right)^{-1} D_{+}^{2} & -P_{-} & 0 & \cdots & 0 \\
0 & -P_{+} & \left(D_{-}^{3}\right)^{-1} D_{+}^{3} & -P_{-} & \ddots & \vdots \\
\vdots & 0 & \ddots & \ddots & \ddots & \vdots \\
0 & \ldots & \vdots & -P_{+}\left(D_{-}^{L_{s}-1}\right)^{-1} D_{+}^{L_{s}-1} & -P_{-} \\
m P_{-} & 0 & \cdots & 0 & -P_{+} & \left(D_{-}^{L_{s}}\right)^{-1} D_{+}^{L_{s}}
\end{array}\right) \text {, }
$$

where $D_{+}^{s} \equiv 1+b_{s} D_{W}\left(-M_{0}\right), D_{-}^{s} \equiv 1-c_{s} D_{W}\left(-M_{0}\right)$ and $P_{ \pm}=\left(1 \pm \gamma_{5}\right) / 2$. Here $b_{s}$ and $c_{s}$ are numerical constants depending on the fifth coordinate $s$. After an unitary transformation and a 
Schur decomposition followed by an introduction of a Pauli-Villars field, one can derive a fourdimensional action $S^{(4)}=\sum_{x} \bar{\psi} D^{(4)} \psi$ with a 4D effective operator

$$
D^{(4)} \equiv \frac{1+m}{2}-\frac{1-m}{2} \gamma_{5} \frac{T_{1}^{-1} T_{2}^{-1} \cdots T_{L_{s}}^{-1}-1}{T_{1}^{-1} T_{2}^{-1} \cdots T_{L_{s}}^{-1}+1}
$$

acting on $4 \mathrm{D}$ fields $\bar{\psi}$ and $\psi$. Here $T$ matrices are defined as $T_{s}^{-1} \equiv-\left(Q_{-}^{s}\right)^{-1} Q_{+}^{-1}$ and $Q_{ \pm}^{s} \equiv$ $\left(D_{-}^{s}\right)^{-1} D_{+}^{s} P_{\mp}-P_{ \pm}$. This gives an operator corresponding to $(\mathbb{L}-\mathbb{C})$ with a sign function approximated by $\varepsilon=\left(1-\prod_{s} T_{s}\right)\left(1+\prod_{s} T_{s}\right)$. The relation between the $5 \mathrm{D}$ and $4 \mathrm{D}$ operators can also be written as

$$
D^{(4)}=\left[\mathscr{P}^{-1}\left(D_{G D W}^{5}(m=1)\right)^{-1} D_{G D W}^{5}(m) \mathscr{P}\right]_{11},
$$

where $D_{G D W}^{5}(m=1)$ plays the role of the Pauli-Villars operator. $\mathscr{P}$ is an projection operator that distributes right-handed and left-handed components of the 4D field to the opposite surfaces of the 5D lattice. The subscript 11 on the right hand side implies that one takes a $4 \mathrm{D}$ block on the upper-left corner of the entire 5D matrix.

By setting the parameters $b_{s}$ and $c_{s}$ as $b_{s}+c_{s}=b \omega_{s}$ and $b_{s}-c_{s}=c$, one can select the kernel and approximation. The kernel $H_{K}$ is given through

$$
T_{s}^{-1}=\frac{1+\omega_{s} H_{K}}{1-\omega_{s} H_{K}}, \quad H_{K}=\gamma_{5} \frac{b D_{W}}{2+c D_{W}} .
$$

The Wilson kernel corresponds to $(b, c)=(2,0)$ while the kernel for the standard domain-wall fermin $H_{T}$ is given by $(b, c)=(1,1)$. By varying $b$ while fixing $c=1$, we obtain the so-called Möbius domain-wall operators [田]. In this work we tested $b=1$ and 2, the latter of which is nearly optimal to minimize the residual mass for $L_{s}$ around 8-12.

The Tanh approximation is obtained by a simple choice $\omega_{s}=1$, with which

$$
\varepsilon\left(H_{T}\right)=\frac{\left(1+H_{T}\right)^{L_{s}}-\left(1-H_{T}\right)^{L_{s}}}{\left(1+H_{T}\right)^{L_{s}}+\left(1-H_{T}\right)^{L_{s}}} .
$$

On the other hand, the rational function approximation can be obtained by choosing $\omega_{s}$ depending on $s$. The optimal choice in a given range of the eigenvalues of $H_{K}$ is that of Zolotarev, which leads to Chiu's optimal domain-wall fermion [5].

For the kernel, there is another option of choosing the link smearing that enters in the WilsonDirac operator $D_{W}$. We apply the stout-link smearing [焑], $N_{s m r}$ times, with $N_{s m r}=0-3$. The smearing parameter $\rho$ is taken to be 0.1 in this work. We confirmed that the near-zero eigenvalues of $H_{W}$ are substantially suppressed [ $[$ ] . It means that a better approximation of the sign function is possible for the same amount of computational cost, and the residual mass is reduced as we will see in the following.

\section{Numerical tests}

We implemented this generalized 5D formulation in our QCD software package Iroiro++, which includes a highly optimized code for IBM Blue Gene/Q [ [ ] ]

Our numerical tests are performed on 30 gauge configurations of a $16^{3} \times 32$ lattice generated with the tree-level Symanzik gauge action and with two-flavors of dynamical fermions. (The details 


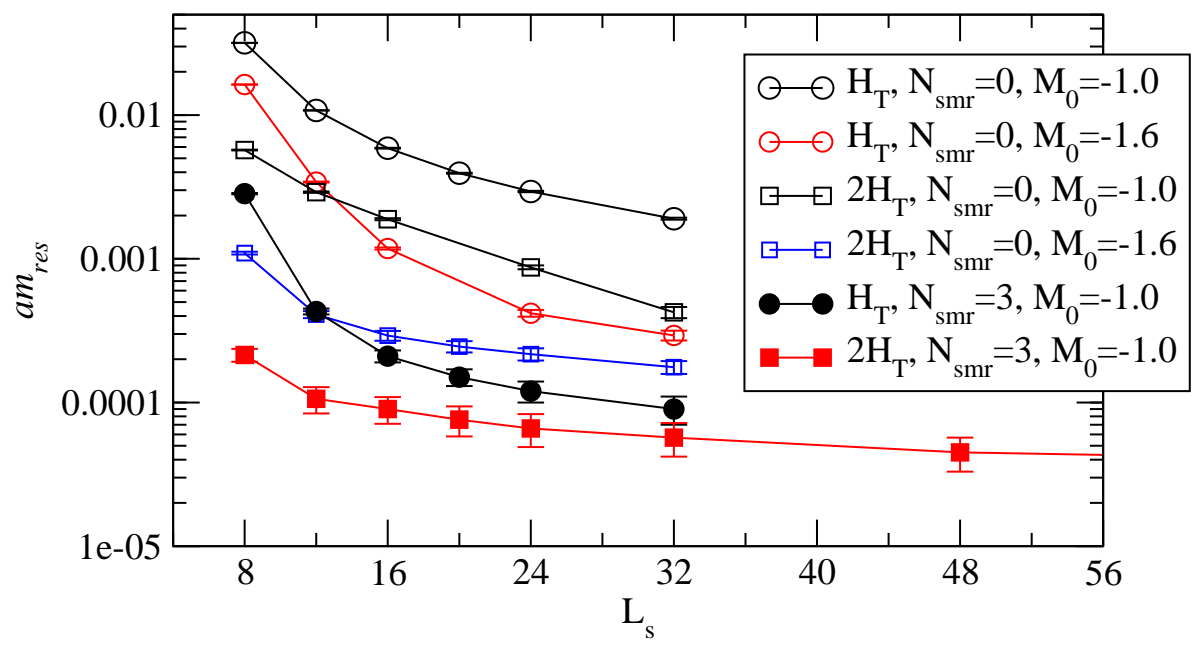

Figure 1: Residual mass with the Tanh approximation is plotted as a function of $L_{s}$. Open (filled) symblos show the data without (with) the smeared link. Circles are those with the conventional Tanh approximation, while those with a scale factor 2 are shown by squares.

of the fermion formulation for the sea quark would not be important for this study.) The lattice spacing determined through the $r_{0}$ scale is about $0.08 \mathrm{fm}$.

We quantify the amount of chiral symmetry violation using the residual mass $m_{\text {res }}$, which is measured as

$$
m_{\text {res }}=\frac{\left\langle\operatorname{tr} G^{\dagger} \Delta_{L} G\right\rangle}{\left\langle\operatorname{tr} G^{\dagger} G\right\rangle},
$$

following $[Q]$. Here $G$ denotes a quark propagator from some source, for which we chose a random noise distributed all over the lattice. The chiral symmetry violation is represented by an operator $\Delta_{L}$ defined through

$$
2 \gamma_{5} \Delta_{L}=\gamma_{5} D^{(4)}+D^{(4)} \gamma_{5}-2 D^{(4)} \gamma_{5} D^{(4)}
$$

which could also be written as $4 \Delta_{L}=1-\operatorname{sgn}^{2}\left(H_{K}\right)$. By construction, it probes the violation of the Ginsparg-Wilson relation. The residual mass calculated using (3. D) has only a minor valence quark mass dependence, which is irrelevant for the purpose of this study. We chose a valence quark mass $a m=0.027$.

\section{1 $m_{\text {res }}$ with Tanh approximation}

Figure $\square$ shows the residual mass calculated for the $H_{T}$ kernels combined with the Tanh approximation. With the simple choice of $H_{T}$ without the smeared link (open circles, black $\left(M_{0}=1.0\right)$ and red $\left(M_{0}=1.6\right)$ ), the residual mass stays relatively large ( $\left.>0.001\right)$ unless $L_{s}$ is set greater than 16 . With the lattice cutoff $2.5 \mathrm{GeV}$ of the configurations used in this study, $m_{\text {res }}=0.001$ corresponds to $2.5 \mathrm{MeV}$, which is small but still non-negligible compared to the physical up and down quark masses $\sim 5 \mathrm{MeV}$. We therefore require the residual mass to be 0.0004 or smaller, which is less than $1 \mathrm{MeV}$. With a scale factor $b=2$ (denoted as $2 H_{T}$ in the plot, blue open squares), this condition is satisfied. Further introducing the link-smearing $N_{s m r}=3$ (filled red squares) we are able to reach $m_{\text {res }}=0.0001$ already at $L_{s}=12$, which corresponds to $0.5 \mathrm{MeV}$ in the physical unit. 


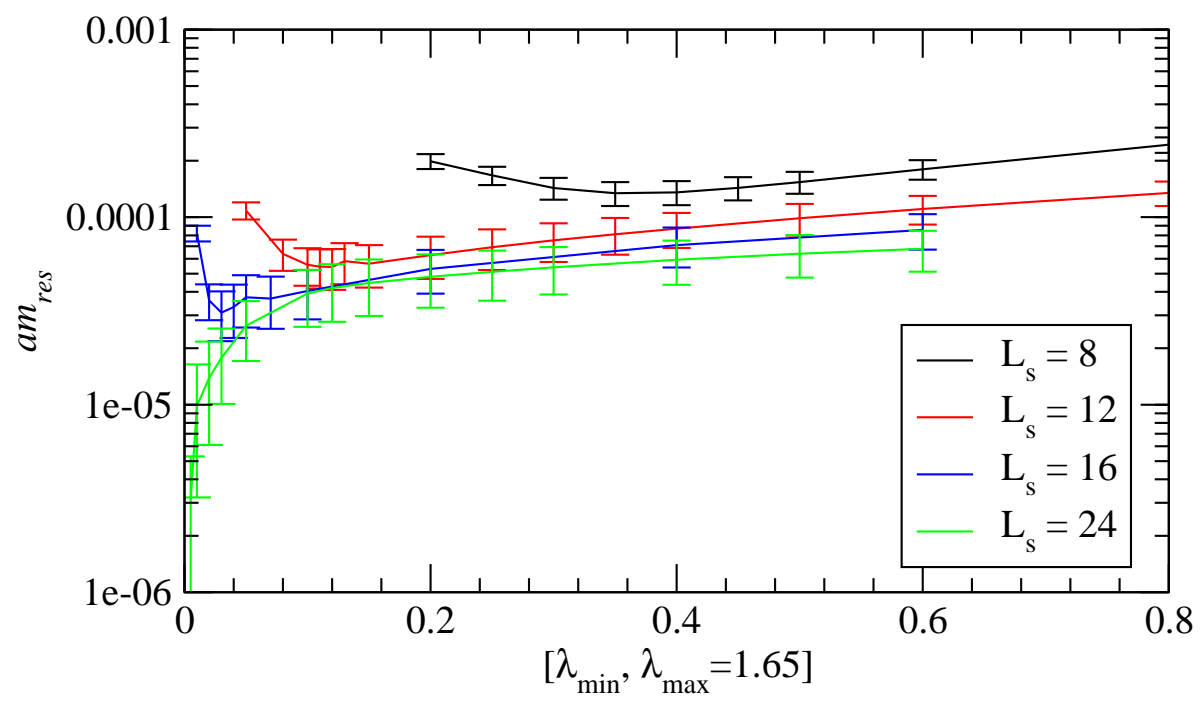

Figure 2: Residual mass with the Zolotarev approximation is plotted as a function of the lower limit $\lambda_{\min }$ of the approximation range $\left[\lambda_{\min }, \lambda_{\max }\right]$, which is applied for the kernel operator $2 H_{T}$. The maximum value $\lambda_{\text {max }}$ is fixed to 1.65. Results are shown for $L_{s}=8,12,16$ and 24 .

\section{2 $m_{\text {res }}$ with Zolotarev approximation}

With the optimal rational approximation (Zolotarev) there are parameters to control the range of the sign function approximation $\left[\lambda_{\min }, \lambda_{\max }\right]$, which is applied for absolute values of the eigenvalues of the kernel operator $H_{K}$. The eigenvalues in this range are approximated to a very good accuracy while those below $\lambda_{\min }$ are significantly away from the sign function.

The plot in Figure $\square$ shows $m_{\text {res }}$ calculated with various values of $\lambda_{\min }$. The kernel is $H_{K}=2 H_{T}$. For a given value of $L_{S}$, which determines the degree of the rational function, there is a value of $\lambda_{\min }$ where $m_{\text {res }}$ is minimized. This is understood because: if $\lambda_{\min }$ is too high, there are more modes out of the range of approximation, while if $\lambda_{\min }$ is too low the approximation becomes less precise over the entire region. Therefore, $m_{\text {res }}$ tends to increase on the both ends.

With $L_{s}=12$ we can achieve $a m_{\text {res }}$ better than 0.0001 , which corresponds to the best choice we found among the Tanh approximations (Figure $\mathbb{D}$ ). This can be achieved without fine tuning of $\lambda_{\text {min }}$.

We obtain very similar results with the Wilson kernel.

\section{Numerical cost}

Naively, the numerical cost for the 5D formulations is proportional to $L_{s}$. There is however an additional significant factor due to the condition number of the 5D matrix that affects the number of conjugate gradient iterations $N_{i n v}$ needed to invert the 5D matrix. Instead of calculating the condition number of the 5D matrix, we simply monitor $N_{i n v}$ for an inversion of $D_{G D W}^{5}(m=1)$, i.e. the Pauli-Villars operator. Then, we use $N_{i n v} L_{s}$ as a measure of the computational cost for each kernel and approximation.

In Figure 3 we compare the cost of the Tanh and Zolotarev approximations for the $2 H_{T}$ kernel. As it should be compared for an equal value of $m_{\text {res }}$, we plot $m_{\text {res }}$ versus $N_{i n v} L_{s}$. Even for a fixed 


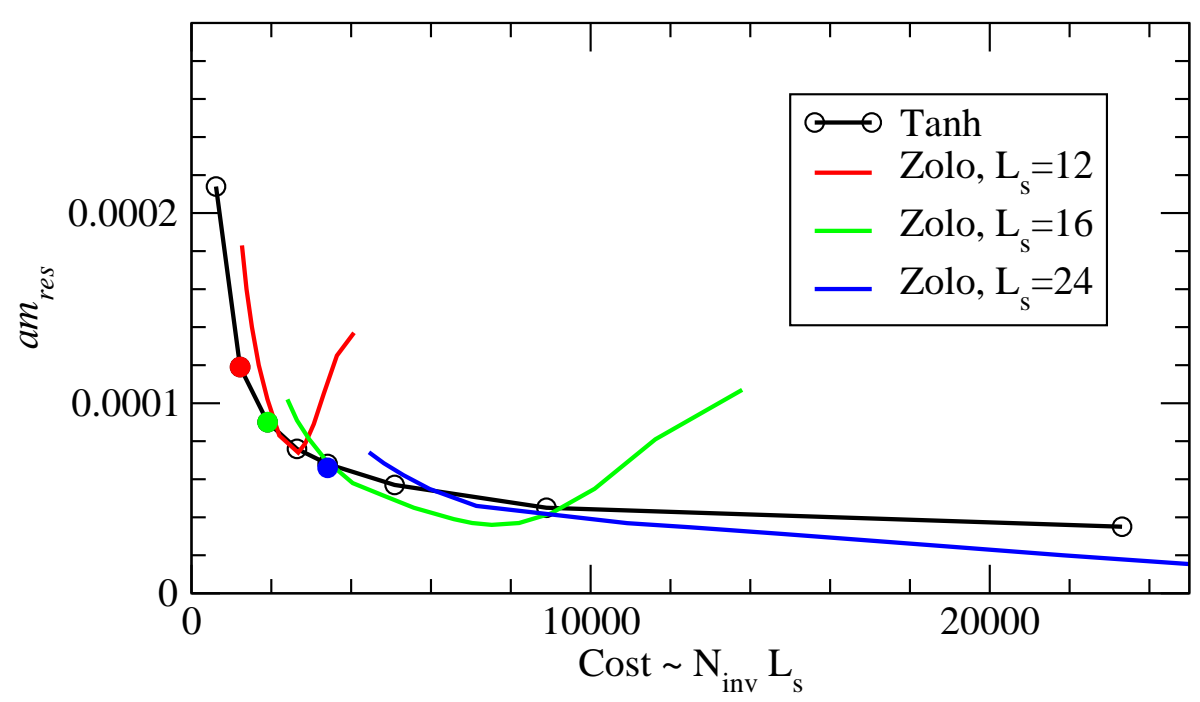

Figure 3: Residual mass plotted as a function of the cost measure $N_{i n v} L_{s}$. Here, $N_{i n v}$ is the number of the conjugate gradient iterations to invert the Pauli-Villars operator $D_{G D W}^{5}(m=1)$, which is required to construct the $4 \mathrm{D}$ effective operator ([2.3). Colored solid curves are the results with the rational function approximation (Zolotarev) with $L_{s}=12$ (red), 16 (green) and 24 (blue). Circles connected by black lines are those with Tanh for various $L_{s}(=8,12,16,20,24, \ldots)$. Points for $L_{s}=8,12$ and 16 are shown with colors corresponding to the Zolotarev curves.

$L_{s}$, the result for the rational function approximation (Zolotarev) varies as large as a factor of 3-5. This is due to the choice of $\lambda_{\min }$, i.e. $N_{i n v}$ rapidly grows as $\lambda_{\min }$ is reduced (in the range indicated in Figure (2). For a fixed $L_{s}$ (in the range of our target $L_{s} \sim 12-24$ ) the cost with Tanh is always lower than Zolotarev (as compared by a dot and a curve of the same color). Roughly speaking, using Zolorarev, $m_{\text {res }}$ can be made as small as a half of that with Tanh, at the cost more than a factor of two more computational cost. Therefore, unless we aim at realizing $m_{\text {res }}$ smaller than $4 \times 10^{-4}$, Tanh is more cost effective.

On the other hand, if we need to achieve $m_{\text {res }} \sim 10^{-4}$, Zolotarev is clearly better, since Tanh cannot reach that level unless $L_{s}$ is as large as 96.

\section{Summary}

This study was initiated to look for the best choice of the nearly chiral lattice fermion action in terms of $m_{\text {res }}$ and numerical cost. In order for $m_{\text {res }}$ to be negligible in the physics analysis, we require $m_{\text {res }} \lesssim 0.5 \mathrm{MeV}$, which is much smaller than the physical up and down quark mass. At the lattice spacing $a \lesssim 0.08 \mathrm{fm}$, this can be achieved with $L_{s}=8$ or 12 using the Tanh approximation with a scale factor $b=2$. In the on-going project by the JLQCD collaboration, this choice is adopted together with the link-smearing $N_{s m r}=3$. A detailed study of the computational cost for the Hybrid Monte Carlo simulation is presented in [ए]], and a feasibility study of reweighting to exactly chiral fermion is found in [प]].

We are currently generating gauge configurations of $2+1$ flavors of sea quarks using the nearly chiral lattice fermion as described above. For the study of heavy flavor physics, fine lattices of $1 / a$ $=2.4,3.6$ and $4.8 \mathrm{GeV}$ are planned on $32^{3} \times 64,48^{3} \times 96$ and $64^{3} \times 128$ lattices. Light quarks are 
taken in the range of pion mass between 220 and $500 \mathrm{MeV}$ keeping the sufficient lattice volume to control the finite volume effect, $m_{\pi} L>4$. So far, we have accumulated our initially targeted statistics on $32^{3}$ lattices and runs are on-going on $48^{3} \times 96$. Physics analysis has just started [ㅁ] ].

Numerical simulations are performed on Hitachi SR16000 M1 and IBM Blue Gene/Q at High Energy Accelerator Research Organization (KEK) under a support of its Large Scale Simulation Program (No. 12/13-04). This work is supported in part by the Grant-in-Aid for Scientific Research (No. 21674002, 25287046, 25800147), the Grant-in-Aid for Scientific Research on Innovative Areas (No. 2004: 20105001, 20105002, 20105003, 20105005, 23105710), and SPIRE (Strategic Program for Innovative Research).

\section{References}

[1] P. H. Ginsparg and K. G. Wilson, Phys. Rev. D 25, 2649 (1982).

[2] H. Neuberger, Phys. Lett. B 417, 141 (1998) [arXiv:hep-lat/9707022].

[3] R. G. Edwards and U. M. Heller, Phys. Rev. D 63, 094505 (2001) [hep-lat/0005002].

[4] R. C. Brower, H. Neff and K. Orginos, arXiv:1206.5214 [hep-lat].

[5] T. -W. Chiu, Phys. Rev. Lett. 90, 071601 (2003) [hep-lat/0209153].

[6] C. Morningstar and M. J. Peardon, Phys. Rev. D 69, 054501 (2004) [hep-lat/0311018].

[7] S. Durr, C. Hoelbling and U. Wenger, JHEP 0509, 030 (2005) [hep-lat/0506027].

[8] G. Cossu, J. Noaki, S. Hashimoto, T. Kaneko, H. Fukaya, P. A. Boyle and J. Doi, PoS LATTICE 2013, 482; arXiv:1311.0084 [hep-lat].

[9] R. G. Edwards, B. Joo, A. D. Kennedy, K. Orginos and U. Wenger, PoS LAT 2005, 146 (2006) [hep-lat/0510086].

[10] T. Kaneko et al. [JLQCD Collaboration], PoS LATTICE 2013, 263; arXiv:1311.6941 [hep-lat].

[11] H. Fukaya et al. [JLQCD Collaboration], PoS LATTICE 2013, 127; arXiv:1311.4646 [hep-lat].

[12] J. Noaki et al. [JLQCD collaboration], PoS LATTICE 2013, 263. 